\title{
Searching for Type Ia Supernova Progenitors
}

\author{
By A L VIO RENZINI
}

\author{
Dipartimento di Astronomia, Università di Bologna, \\ CP 596, I-40100 Bologna, Italy \\ and European Southern Observatory
}

The status for the identification of specific astronomical objects as SNIa progenitors is reviewed. Single or double degenerate progenitors? Chandrasekhar or sub-Chandrasekhar mass exploders? These are the two main questions still to be answered concerning the progenitors of Type Ia supernovae. Although all four combinations may be represented in nature, searches for double degenerates seem to indicate that such systems provide a minor channel for the production of SNIa's. The more promising candidates appear to be symbiotic stars, consisting of a single degenerate star and a sub-Chandrasekhar mass star.

\section{Introduction}

The nature of the progenitors of Type Ia SNe remains highly conjectural. The fact that SNIa's occur in elliptical galaxies - where star formation ceased a very long time ago - indicates that at least in some cases there is a long delay between the formation of the progenitor and the explosion. Attention has generally concentrated on white dwarfs (WD) in binary systems, in which the explosion of the WD is triggered by accretion from the companion. Various WD explosion mechanisms are discussed by Ken'ichi Nomoto and Eli Livne at this meeting, and I will here deal with the identification of specific astronomical objects as suitable precursor candidates.

The two main questions are schematically represented in Fig. 1. In the SD model (Whelan \& Iben 1973) the companion to the WD is a living, evolved star pouring matter onto the WD. In the DD model (Iben \& Tutukov 1984; Webbink 1984; Paczyński 1985) the companion is also a WD, and the explosion occurs when the two WDs merge as a result of the angular momentum losses via gravitational wave radiation (GWR). In the SD model only a thermonuclear clock (i.e. the secondary's lifetime) sets the explosion time, while in the DD model also the GWR clock is at work.

In the classical scenario the explosion takes place when the mass of the accreting WD reaches the Chandrasekhar limit $\left(\sim 1.4 M_{\odot}\right)$, and carbon is ignited under extremely degenerate conditions. However, reaching the Chandrasekhar limit is not a necessary condition for a WD to explode. Helium detonations and double (He and C) detonations are indeed possible in SD models provided that the accretion rate is in a suitable range (see Nomoto, these proceedings), which may be the case for $\mathrm{C} / \mathrm{O}-\mathrm{WD}+\mathrm{He}-\mathrm{WD}$ pairs in DD models. Four basic combinations of the two pairs of options are therefore possible, as sketched in Fig. 1. The identification of SNIa progenitors consists primarily in determining the relative weight of the four basic combinations: DD/Ch, SD/Ch, DD/Sub-Ch, and SD/Sub-Ch, all of which might be represented in nature. Such determination would be important for a number of astrophysical problems. In particular, it would help us to figure out how the SNIa rate evolves past a generic burst of star formation, i.e., its rise time to the maximum rate, and the timescale of the subsequent decline. This would be especially relevant for the determination of the rate of iron release in galaxies and clusters of galaxies (see Renzini et al. 1993 and references therein), and for the evolution of the SN heating of the interstellar medium, in particular in the case of elliptical galaxies (e.g., Ciotti et al. 1991). 


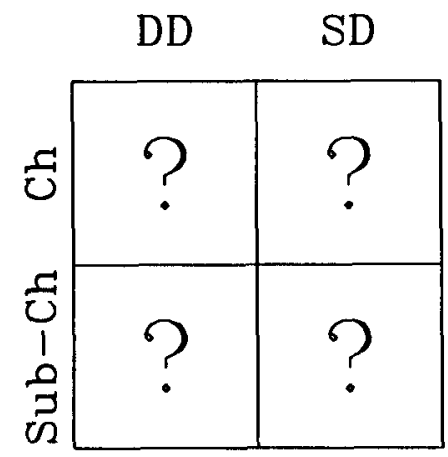

Figure 1. The SN Ia Dilemma: Single (SD) or Double Degenerate (DD)? Chandrasekhar (Ch) or Sub-Ch Mass Exploders?

\section{The Double Degenerate Scenario and the Search for DD Binaries}

\subsection{Binary Evolution Leading to DD Systems}

There is little doubt that DD systems exist, as a large fraction of stars are born as member of a binary, and most stars will leave WD remnants. The question is how hard are the DD systems at their formation, when the secondary star also becomes a WD. Once formed, a DD system will merge via GWR losses in a time:

$$
t_{\mathrm{GWR}}(\mathrm{yr})=\frac{1.5 \times 10^{8} A_{\mathrm{ff}}^{4}}{M_{1 \mathrm{R}} M_{2 \mathrm{R}}\left(M_{1 \mathrm{R}}+M_{2 \mathrm{R}}\right)} \simeq \frac{8 \times 10^{7} P^{8 / 3}\left(M_{1 \mathrm{R}}+M_{2 \mathrm{R}}\right)^{1 / 3}}{M_{1 \mathrm{R}} M_{2 \mathrm{R}}},
$$

where $A_{\mathrm{ff}}$ is the separation in $R_{\odot}$ units, $P$ its period in hours, and the mass of the two WDs is in $M_{\odot}$ units. The problem is to determine how the initial binary parameters $M_{1}, M_{2}$, and $A_{\circ}$ map into the corresponding final values $M_{1 \mathrm{R}}, M_{2 \mathrm{R}}$, and $A_{\mathrm{ff}}$, when the secondary star also becomes a WD. Thus, the merging rate - and the SNIa rate - both depend on (1) the distribution of the initial binary parameters; and (2) how they map into the corresponding final values. The answer to (2) depends on how much mass and angular momentum have been lost during the binary evolution through two common envelope events (CEE), resulting from the Roche lobe filling of the primary and of the secondary component, respectively. As for other hydrodynamical processes in stellar physics (e.g., convection and mass loss) a reasonable parameterization is currently unavoidable to describe the outcome of CEEs. In this mood, the so-called $\alpha$-parameter (Tutukov \& Yungelson 1979) has been generally adopted, where $\alpha$ is implicitly defined by:

$$
G M_{1}^{2} / A_{\circ}=\alpha G M_{1 \mathrm{R}} M_{2} / A_{\mathrm{f}}
$$

where $A_{\mathrm{f}}$ is the binary separation after the first CEE. Seemingly:

$$
G M_{2}^{2} / A_{f}=\alpha G M_{1 \mathrm{R}} M_{2 \mathrm{R}} / A_{\mathrm{ff}},
$$

for the second CEE. The dimensionless parameter $\alpha$ is thus a measure of the effectiveness of the transformation of the orbital energy into work to eject the CE material from the system. For small $\alpha(\ll 1)$ the transformation is inefficient, and considerable orbital shrinkage results; for large $\alpha(\gtrsim 1)$ the orbital shrinkage is very modest, and an energy source other than orbital is required to drive the $\mathrm{CE}$ off. According to $2 \mathrm{D}$ and $3 \mathrm{D}$ hydrodynamical studies (Livio 1989; Taam \& Bodenheimer 1989), $\alpha$ could be rather small $(\simeq 0.3-0.6)$, but the current state of the art in these simulations leaves ample room for $\alpha \gtrsim 1$.

Unfortunately, $t_{\mathrm{GWR}}$ is extremely sensitive to $\alpha$ (actually, $t_{\mathrm{GWR}} \propto \alpha^{9}$, Iben \& Webbink 
1989). For example, a binary with $\alpha=1$ would produce a DD that merges in one Hubble time ( $\sim 15 \mathrm{Gyr}$ ), while one with $\alpha=0.3$ would instead generate a DD merging in only $\sim 3 \times 10^{5} \mathrm{yr}$. So, a factor of $\sim 3$ uncertainty in $\alpha$ means a factor $\sim 3^{9} \simeq 2 \times 10^{4}$ uncertainty in the lifetime of the DD systems, which translates into a large uncertainty in their predicted local density. [Such density does not scale precisely as $\alpha^{9}$ (or as $\alpha_{1}^{5} \alpha_{2}^{4}$, if $\alpha_{1} \neq \alpha_{2}$ ) thanks to a compensating effect, as changing $\alpha$ what changes is the range of initial separations that produce DDs with $t_{\mathrm{GWR}}$ in a certain range.] Only the case $\alpha=1$ has been fully explored so far (Iben 1990; Tutukov \& Yungelson 1992; Tutukov, Yungelson, \& Iben 1992), but the very large dependence of $t_{\mathrm{GWR}}$ on this poorly known parameter makes binary evolution theory unable to predict with any degree of confidence the number, period distribution, and merging rate of DD systems. Turning the problem around, one can say that the extreme sensitivity of $t_{\mathrm{GWR}}$ to $\alpha$ implies that suitable observations may set tight limits on $\alpha$. This is the point of view adopted in the next section.

\subsection{Searching for DD Systems}

DD systems that can merge in less than one Hubble time $\left(t_{\mathrm{GWR}} \lesssim 15 \mathrm{Gyr}\right)$ would have sufficiently large orbital velocity ( $v_{\mathrm{ORB}} \gtrsim 300 \mathrm{~km} \mathrm{~s}^{-1}$ (with orbital periods $P \lesssim 10^{\mathrm{h}}$ ) that it should be fairly easy to discover them as spectroscopic binaries. Three systematic surveys of WDs have been undertaken to hunt for DDs, in particular those that could be the precursors of SNIa events. The surveys are all based on looking for radial velocity or line shape variations, though with different sensitivities.

First, Robinson and Shafter (1987, hereafter RS) have completed a survey of 44 WDs (40 DAs and $4 \mathrm{DBs}$ ), but with a null result. Their technique allowed only the detection of very short period systems $\left(P \lesssim 3^{\mathrm{h}}\right)$. Second, Foss, Wade, \& Green (1991) have surveyed 25 DA WDs. Their survey was also sensitive to somewhat longer periods $\left(P \lesssim 10^{\mathrm{h}}\right)$, but again they found null results. Finally, Bragaglia et al. (1990; in prep.) have so far scrutinized 84 WDs (54 DAs and $30 \mathrm{DBs}$ ). They found one DD (with $P=1^{\mathrm{d}} .15$ ) and identified 3 other DD candidates among the DAs and 6 candidates among the DBs. "Candidates" are here defined as those WDs for which at least two spectra give radial velocities differing by more than $\sim 100 \mathrm{~km} \mathrm{~s}^{-1}(\mathrm{a} \sim 2.5 \sigma$ effect $)$. This survey was sensitive up to $P \approx 3^{\mathrm{d}}$.

In order to check the DD scenario RS proceeded as follows:

1) They assumed that all DDs in the WD sample, with $P<P_{\max }\left(=3^{\mathrm{h}}\right)$, were detected.

2) They assumed that all DDs form with $P>P_{\max }$, and then drift in period via GWR until they merge.

3) They estimated the space density of DDs required to account for the observed SNI rate in the Galaxy, and compared their result with the observed limit.

From their null result RS estimated an upper limit to the observed space density of DDs. Their result, $N_{\mathrm{DD}}\left(P<3^{\mathrm{h}}\right)_{\mathrm{obs}} \lesssim 3 \times 10^{-5} \mathrm{pc}^{-3}$, compares to the value $N_{\mathrm{DD}}(P<$ $\left.3^{\mathrm{h}}\right)_{\text {req }} \simeq 3 \times 10^{-5} \mathrm{pc}^{-3}$ required to account for the SNI rate. RS then conclude that DDs are unlikely to be the precursors of SNIs, unless assumption (2) is wrong, i.e., unless most of DDs form initially with $P<P_{\max }$. Indeed, though the two numbers appear to be marginally consistent, one should consider that most DDs will have a combined mass $\lesssim 1.4 M_{\odot}$, and are not suitable SN precursors.

To derive the required DD space density, RS used a SNI rate $0.02 \mathrm{yr}^{-1}$ (Tammann 1982), a value considerably larger than currently estimated for SNIa's. Indeed, in the old rate all the SNI subtypes were lumped together, while the DD conjecture is supposed to apply only to SNIa's. Thus, with the current Galactic SNIa rate of $0.003 \mathrm{yr}^{-1}$ (van den Bergh \& Tammann 1991), the required space density drops to $N_{\mathrm{DD}}\left(P<3^{\mathrm{h}}\right)_{\text {req }} \simeq$ $4 \times 10^{-6} \mathrm{pc}^{-3}$ and the RS survey alone cannot set a strong constraint on the DD scenario. 


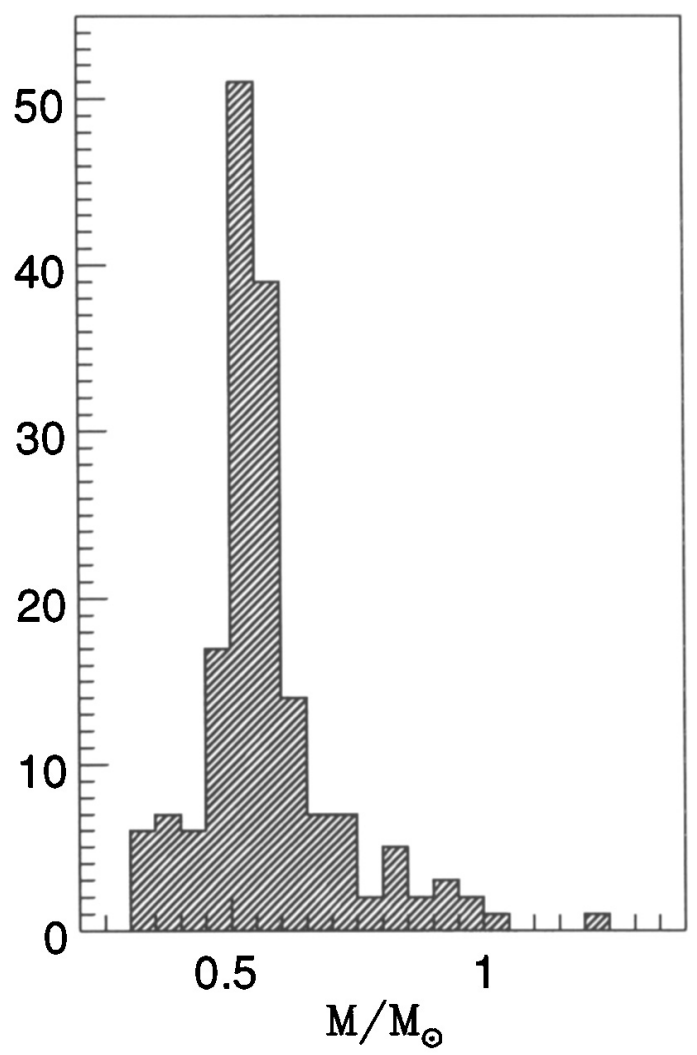

FIGURE 2. The mass distribution for 170 WDs of the DA subtype from the combined samples of Bergeron, Saffer, \& Liebert (1992) and Bragaglia et al. (1990). The individual masses are estimated from surface gravity via Balmer line fitting (Bragaglia, Renzini, \& Bergeron 1993)

Even combining all the three surveys (which gives a total of 118 WDs, none of which is a DD with $\left.P<3^{\mathrm{h}}\right)$ one finds that the upper limit, $N_{\mathrm{DD}}\left(P<3^{\mathrm{h}}\right)_{\mathrm{obs}} \lesssim 1 \times 10^{-5} \mathrm{pc}^{-3}$, is still a factor $\sim 2.5$ above the required value.

However, combining the Bragaglia et al. sample of DA WDs with the Foss et al. sample one has a total of $89 \mathrm{WDs}$, none of which is a DD with $P<10^{\mathrm{h}}$. From this I estimate $N_{\mathrm{DD}}\left(P<10^{\mathrm{h}}\right)_{\text {obs }} \lesssim 1.5 \times 10^{-5} \mathrm{pc}^{-3}$, which compares to $N_{\mathrm{DD}}\left(P<10^{\mathrm{h}}\right)_{\mathrm{req}} \simeq$ $8.7 \times 10^{-5} \mathrm{pc}^{-3}$. This last estimate implies that about a dozen out of 89 WDs should be DDs with $P<10^{\text {h }}$; but none is found! We must conclude that DDs are NOT the SNIa progenitors, unless RS assumption 2) is violated - i.e., most DDs should form with $P<10^{\mathrm{h}}$, and then $<t_{\mathrm{GWR}}>\ll 10^{10} \mathrm{yr}$. One can estimate that most DDs should form with $P \lesssim 5^{\text {h }}$, i.e., there must be a lot of orbital shrinkage through the two CEEs for the DD option to be viable for Galactic SNIa. This scenario would require a rather small $\alpha$ (say 0.3-0.4). Still, there might be a problem with the SNIa's in ellipticals, for which a delay of order of one Hubble time is required.

masses are estimated from surface gravity via Balmer line fitting (Bragaglia, Renzini, \& Bergeron 1993).

The Bragaglia,et al. survey has still more to say - but now for what it did find, rather than for what it did not find. The confirmed and candidate DDs in their sample have all orbital velocities $K \lesssim 100 \mathrm{~km} \mathrm{~s} s^{-1}$. The same applies to a group of low mass $\left(\lesssim 0.4 M_{\odot}\right)$ WDs shown in Fig. 2, which are also likely to be in DD binaries. Indeed, only Roche 
lobe overflow could produce such low mass helium WDs. Including the DD studied by Saffer, Liebert, \& Olzewski 1988), we have a total of 15 helium WDs which are confirmed or very likely DD members, all with $K \lesssim 100 \mathrm{~km} \mathrm{~s}^{-1}$. This result is at variance with the expectation for the $\alpha=1$ case (Iben 1990), which predicts a nearly equal number of DDs with $K \lesssim 100 \mathrm{~km} \mathrm{~s}^{-1}$ as with $100 \lesssim K \lesssim 500 \mathrm{~km} \mathrm{~s}^{-1}$. Moreover, most WDs in the Bragaglia et al. survey are rather bright, hot, most likely young WDs, which should have suffered little GWR orbital decay up to now. Thus, CEEs appear to result in little orbital shrinkage, and a large value of $\alpha(\gtrsim 1)$ seems to be needed in order to reconcile the theoretical expectations with the observed distribution of DD orbital velocities (Bragaglia, Greggio, \& Renzini 1991).

In summary, the observed DD option for the SNIa precursors requires $\alpha$ to be small, while instead the properties of the observed helium WDs in DD systems demand $\alpha$ to be large. Since I did not find any clever way of reconciling these two opposite requirements, I am forced for the time being to conclude that the prognosis for the DD scenario does not appear good. It simply appears that binaries evolving in isolation may not lose enough angular momentum to produce hard DDs able to merge in one Hubble time or less. This conclusion does not necessarily imply that DD merging does not take place in nature. In a very high density environment - such as in dense globular clusters and in galactic nuclei - angular momentum removal by encounters with other stars may well lead to very hard DDs, with separation $\lesssim 3 R_{\odot}$, which are then able to merge in a relatively short time. Perhaps this is the main channel for DD merging, and its outcome - rather than a SNIa - may lead to accretion induced collapse (Nomoto 1988) and the subsequent formation of millisecond pulsars now so copiously found in globular clusters (Grindlay \& Baylin 1988).

It is worth emphasizing that the above considerations refer primarily to WDs of the DA variety (those retaining a thin hydrogen envelope). Actually, there are reasons to believe that massive carbon/oxygen WDs in DD systems are likely to have completely lost their hydrogen envelope, thus disguising themselves as non-DA WDs (Bragaglia et al. 1991). The above calculations should then be repeated for the sample of $30 \mathrm{DBs}$ so far observed. Of them none is a DD with a period shorter than about one day - which may already say something - but a larger sample would be required before reaching firm conclusions.

The last point concerns the immediate SNIa progenitors. The merging of the two WDs leads to the formation of a thick accretion disk around the more massive WD, leading to an object sometimes depicted as a kind of flying saucer (Iben \& Tutukov 1984). However, the final fate of the flying saucer, - even if its mass exceeds $1.4 M_{\odot}$ - is not necessarily a SNIa. This would happen only if the accretion disk has a long enough lifetime, so as to pour slowly its material onto the WD. Otherwise a neutron star would result, rather than a SNIa explosion (Nomoto \& Iben 1985). The accretion process should last $\sim 10^{6}$ yr for a good explosion to occur. From the Galactic SNIa rate one immediately derives that a few thousand such flying saucers should be wandering around the Galaxy. In order to radiate away the potential energy of the thick disk in $\sim 10^{6} \mathrm{yr}$, their typical UV luminosity should be $100-1000 L_{\odot}$. No such object has been found yet.

\section{The Single Degenerate Scenario and SD Candidates}

The other way to let WDs to grow is to feed them ( $\mathrm{H}$ or $\mathrm{He}$ ) fuel, process it (respectively to $\mathrm{He}$ and $\mathrm{C}+\mathrm{O}$ ), until either helium or carbon ignite explosively. This SD scenario comes in two main options, depending on whether companion of the WD is: (1) a main sequence (MS) star; or (2) a red giant (RG) star. Mass transferring WD+MS pairs are usually cataclysmic variables (CV), such as classical novae. The problem with CVs as SNIa 
progenitors is that the WD may not grow at all (most of the accreted material should be ejected during nova outbursts), so that too few such systems may eventually manage to explode and produce a SNIa display (Livio \& Truran 1992). This leaves WD+RG pairs, objects that are known as symbiotic stars (SS) when mass transfer is active.

SSs possess several attractive properties as SNIa progenitors: (1) the luminosity of the accreting WD is typically in the range $10^{2} \lesssim L \lesssim 10^{4} L_{\odot} ;(2)$ the accreted H-rich material appears to being burned quasi-statically (no mass ejection such as in nova outburst is taking place); and (3) the observed luminosities - if due to stationary H-burning - imply accretion and burning rates in the range $10^{-9} \lesssim \dot{M}_{\mathrm{acc}} \lesssim 10^{-7} M_{\odot} \mathrm{yr}^{-1}$ or more (see e.g., Mürset et al. 1991; Munari \& Renzini 1992, hereafter MR). Such values of the accretion rate are indeed very attractive, as they avoid the problem of nova outburst and the associated mass ejection and so allow the WD to grow until explosion (see e.g., Nomoto 1986). The traditional argument against SSs as SNIa progenitors is that there are be too few of them to account for the observed rate (e.g., Wheeler 1991). Supposing that each WD must accrete $0.4 M_{\odot}$ before making a SNIa and that $\dot{M}_{\text {acc }} \simeq 10^{-8}-10^{-7} M_{\odot \mathrm{yr}^{-1}}$, we find that the SS lifetime should be in the range $\tau_{\mathrm{SS}} \simeq 0.4 / \dot{M}_{\mathrm{acc}} \simeq 4 \times 10^{6}-4 \times 10^{7} \mathrm{yr}$. With a Galactic SNIa rate $0.003 \mathrm{yr}^{-1}$, the number os SSs in the Galaxy - precursors to SNIa events - should correspondingly be $0.003 \times \tau_{\mathrm{SS}} \simeq 10^{4}-10^{5}$. This compares to an estimate of only 3000 symbiotics in the whole Galaxy by Kenyon (1986), extrapolating from the 150 known SSs.

$\mathrm{MR}$ have recently reassessed the SS distance scale, population assignment (bulge/old disk rather than young disk), and discovery probability. The first step suggests an increase by a factor $\sim 6$ in the number of Galactic SSs, the second, another factor $\sim 3$, and the third, still another factor $\sim 5$. From these corrections, MR conclude that the Galactic population of SSs may well amount to $\sim 300,000$ objects, some 100 times more than estimated before. If so, SSs could be resurrected as viable SNIa progenitors. With a typical SS lifetime of $\sim 4 \times 10^{6} \mathrm{yr}$, such a population gives a galactic SS formation rate $\sim 0.07 \mathrm{yr}^{-1}$. Is this a reasonable number? Using the luminosity-number relation (Renzini \& Buzzoni 1986), we estimate that the rate of red giant formation in the Galactic bulge + old disk (nearly equal to the corresponding stellar death rate) should be $B(t) L_{\mathrm{T}} \simeq$ $2 \times 10^{-11} \times 3 \times 10^{10} \simeq 0.6$ stars $\mathrm{yr}^{-1}$, where $B(t)$ is the specific evolutionary flux and $L_{\mathrm{T}}$ is the total luminosity of the bulge + old disk. With a lifetime on the upper red giant branch $(\mathrm{RGB}) \sim 10^{8} \mathrm{yr}$, the galactic population of upper RGB stars should be $\sim 0.6 \times 10^{8}=6 \times 10^{7} \mathrm{RGB}$ stars. Thus, the MR estimate requires that just 1 every 200 RGB stars is a symbiotic, which seems quite reasonable.

Kenyon et al. (1993, hereafter KLMT) do not share this optimism. They first adopt a rate of binary formation $r_{\mathrm{bin}} \simeq \frac{1}{2} r_{\mathrm{PN}}=0.4 \mathrm{yr}^{-1}$, where $r_{\mathrm{PN}}$ is the Galactic formation rate of planetary nebulae (almost identical to the Galactic death rate), and then introduce a Drake-like equation for the SS formation rate:

$$
r_{\mathrm{SS}}=r_{\mathrm{bin}} f_{1} f_{2} f_{3}=\frac{1}{2} r_{\mathrm{PN}} f_{1} f_{2} f_{3}
$$

where $f_{1}$ is the fraction of binaries with initial period in the range between 1 and $10 \mathrm{yr}$ (with periods out of this range binaries do not go through a SS phase), $f_{2}$ is the fraction of binaries that survive the first CEE with still a long period to allow the secondary to become a red giant, and $f_{3}$ is the fraction of binaries with a secondary massive enough to evolve to the RGB in less than 15 Gyr. Adopting $f_{1} \simeq 0.11, f_{2} \simeq 0.5$, and $f_{3} \simeq 0.3$, KLMT then estimate $r_{\mathrm{SS}} \simeq 0.007$, i.e. $\sim 10$ times less than the MR result. The resulting Galactic population of $\sim 30,000$ symbiotics -10 times than previously estimated by Kenyon (1986) - is still 10 times less than claimed by MR. Kenyon et al. conclude that 
for the MR estimate to be correct, a value $f_{2} \approx f_{3} \approx 1$ is required; they consider such an assumption "unduly optimistic".

I maintain, however, that both factors are - by definition - equal to unity $\left(f_{2} \equiv f_{3} \equiv 1\right)$. Indeed, $f_{3} \equiv 1$ because eq. (4) relates the SS formation rate to the single star death rate (not the birth rate!) and so already accounts for the fact that only a fraction of formed stars are now evolving to death. So KLMT have mistaken death for birth. Concerning $f_{2}$, KLMT justify their assumption with the need to allow space for the production of CVs. Yet, CVs are mostly WD+MS pairs, and the dwarf fills its the Roche lobe because of magnetic wind orbital decay rather than by swelling to red giant size (Verbunt \& Zwaan 1981). Thus a different clock is at work in the case of CVs, and their production rate cannot be set proportional to the single star death rate, whose clock is exclusively nuclear. With $f_{2}=f_{3}=1$ the discrepancy between the two estimates drops from a factor of 10 down to less than a factor of 2 , certainly within the reasonable uncertainty in this kind of evaluation. In conclusion, with a Galactic single star death rate $\sim 1 \operatorname{star}^{\mathrm{yr}^{-1}}$, a SS formation rate $\sim 0.07 \mathrm{yr}^{-1}$, and a SNIa rate $\sim 0.003 \mathrm{yr}^{-1}$ we find that approximately one every 10 stars should become a symbiotic, and one of every 20 SS should eventually become a SNIa. These round numbers sound quite reasonable to me.

On one point made by KLMT I definitely agree: SSs become more likely SNIa progenitors if the accreting WD could explode before reaching $1.4 M_{\odot}$, as in helium detonation and double detonation models. KLMT make the point that in this case less material need be accreted to produce the explosion. Perhaps more importantly, in this way not only the most massive WDs (with $M_{\mathrm{WD}} \gtrsim 1 M_{\odot}$ ) are likely SNIa progenitors (see Fig. 2 how few of them there are!), but objects in the main body of the WD mass distribution immediately become potential progenitors.

Along with arguments supporting the SD scenario, it is worth mentioning at least some of the counterarguments. The absence of hydrogen lines in SNIa spectra has been a traditional point against the SD scenario, though Balmer line emission is not expected to appear before the nebular stage of the supernova. MR mention two known cases with hints of hydrogen lines, and Ruiz-Lapuente et al. now report at this meeting a third case (SN1991bg). Systematic observations of SNIa's through the nebular stage would then be very important. The absence of radio emission in SNIa's argues against the existence of a (massive) circumstellar envelope around SNIa precursors, while SSs should be embedded in some circumstellar material. A great difference is expected, however, between the strong winds typical of massive red supergiants (the progenitors of SNII's), which have mass loss rates $10^{-6}-10^{-5} M_{\odot} \mathrm{yr}^{-1}$, and the weaker winds of SSs, which have $\left(\dot{M} \lesssim 10^{-8}-10^{-7} M_{\odot} \mathrm{yr}^{-1}\right)$.

For every scenario, explaining SNIa's in ellipticals is perhaps the most demanding aspect of the problem, and the SD option is no exception. RGB stars in ellipticals have a mass of $\sim 0.85 M_{\odot}$, of which $\gtrsim 0.25 M_{\odot}$ are locked into the degenerate helium core and are not available for transfer. This leaves a reservoir of only $\sim 0.6 M_{\odot}$. Assuming to start with one of the most massive WDs, with $M_{\mathrm{WD}} \simeq 1 M_{\odot}$, then $\sim 0.4 M_{\odot}$ needs to be accreted in order to reach the Chandrasekhar limit, requiring mass transfer with efficiency exceeding $\sim 66 \%$. Somewhat less dramatic is the case of Sub-Ch exploders, where the WD needs to accrete $\sim 0.2 M_{\odot}$ to lead to a helium detonation (Limongi \& Tornambè 1991; Woosley \& Weaver 1994). Now the efficiency needs to be only $\sim 30 \%$, still high, but not high enough to argue in favor of the Sub-Ch option.

It is quite doubtful that accretion from a Roche-lobe filling companion - or from a wind - may reach such high transfer efficiencies. MR suggest a possible way out from this difficulty, noting that the SS red giant member most often does not fill its Roche lobe, while the mass transfer rate appears to exceed the mass loss rate from a single 
red giant of the same luminosity and spectral type. MR propose that the soft X-ray and UV radiation from the bright accreting WD may stimulate mass extraction from the heated, facing lobe of the red giant, thus leading to a self-regulating mass transfer process. Stimulated mass transfer of this kind has been studied in the context of low mass X-ray binaries, with neutron star + MS and NS + WD pairs. Hydrodynamical simulations show that the velocity of the stimulated wind is generally only a fraction of the escape velocity from the system (Tavani \& London 1993). If this situation applies also to WD $+\mathrm{RG}$ pairs, then a fraction of the extracted material will be accreted by the WD, a fraction may fall back on the RG (thus remaining available for subsequent transfer), and a fraction may escape from the outer Lagrangian point and be lost. Observations as well as hydrodynamical simulation may test this suggestion, along with the very intriguing possibility that - when the accretor is a compact object - stimulated mass transfer may prevent, and then suppress the second CEE. If true, this hypothesis would call for a major revision of our current views on binary evolution. After all, what compelling evidence do we have that binaries experience two - not just one - CEEs?

All in all, of the four options in Fig. 1, the SD/Sub-Ch combination scores somewhat better than the others. Schematically enough, SSs exist while short period DDs are yet to be found. Moreover, the accretion of $\sim 0.2 M_{\odot}$ onto a $\sim 0.6-0.8 M_{\odot}$ WD must certainly a more frequent event than the accretion of $0.4 M_{\odot}$ onto a $\sim 1 M_{\odot}$ WD. If reaching the Chandrasekhar limit is really necessary for SNIa's, what other fate can there be for the much more frequent helium detonators?

\section{Acknowledgements}

I am deeply indebted with Angela Bragaglia, Laura Greggio, and Ulisse Munari for the joint work on this subject, and I am grateful to Marco Tavani and Amedeo Tornambè for very useful discussions.

\section{REFERENCES}

Bergeron, P., Saffer, R. A., and Liebert, J. 1992, ApJ, 394, 228

Bragaglia, A., Greggio, L., Renzini, A., \& D'Odorico, S. 1990, ApJ, 365, L13

Bragaglia, A., Greggio, L., Renzini, A., \& D'Odorico, S. 1991, in Supernovae, ed S.E. Woosley (Berlin: Springer-Verlag), p. 599

Bragaglia, A., Greggio, L., Renzini, A. 1991, in SN1987A and Other Supernovae, ed. I.J. Danziger \& K. Kjar (Garching: ESO), p. 47

Bragaglia, A., Renzini, A., \& Bergeron, P. 1993, in White Dwarfs, ed. M. Barstow (Dordrecht: Kluwer), in press

Ciotti, L., D'Ercole, A., Pellegrini, S., \& Renzini, A. 1991, ApJ, 376, 380

Foss, D., Wade, R.A., \& Green, R. F. 1991, ApJ, 374, 281

Grindlay, J. E., Baylin, C. D. 1988, Nature, 336, 48

Iben, I., Jr. 1990, ApJ, 353, 215

Iben, I., Jr., \& Tutukov, A. V. 1984, ApJS, 54, 335

Iben, I., Jr., \& Webbink, R. F. 1989, in White Dwarfs, ed. G. Wegner (Berlin: Springer-Verlag), p. 377

Kenyon, S. J. 1986, The Symbiotic Stars (Cambridge Univ. Press)

Kenyon, S. J., Livio, M., Mikołajewska, J., \& Tout, C. A. 1993, ApJ, 407, L81 (KLMT)

Limongi, M., \& Tornambè, A. 1991, ApJ, 371, 317

Livio, M. 1989, Space Sci. Rev., 50, 299. 
Livio, M., \& Truran, J. W. 1992, ApJ, 389, 695

Munari, U., \& Renzini, A. 1992, ApJ, 397, L87 (MR)

Mürset, U. Nussbauber, H., Schmidt, H. M., \& Vogel, M. 1991, A\&A, 248, 458

Nomoto, K. 1986, Progress in Particle and Nuclear Physics, 17, 249

Nomoto, K. 1988, in Origin and Evolution of Neutron Stars, ed. D. Helfand \& J. Huang (Dordrecht: Reidel), p. 281

Paczyǹski, B. 1985, in Cataclysmic Variables and Low Mass X-ray Binaries, ed. D. Q. Lamb \& J. Patterson (Dordrecht: Reidel), p. 1

Renzini, A., \& Buzzoni, A. 1986, in Spectral Evolution of Galaxies, ed. C. Chiosi \& A. Renzini (Dordrecht: Reidel), p. 135

Renzini, A., Ciotti, L., D'Ercole, A., \& Pellegrini, S. 1993, ApJ, 419, 52

Robinson, E. L., \& Shafter, A. W. 1987, ApJ, 332, 296 (RS)

Saffer, R.A., Liebert, J., \& Olszewski, E.W. 1988, ApJ, 334, 947

Taam, R.E., \& Bodenheimer, P. 1989, ApJ, 337, 849

Tavani, M., \& London, R. 1993, ApJ, 410, 281

Tutukov, A. A., \& Yungelson, L.R. 1979, in Mass Loss and Evolution of O-Type Stars, ed. P.S. Conti \& C.W.H. De Loore (Dordrecht: Reidel), p. 401.

Tutukov, A. A., \& Yungelson, L. R. 1992, Soviet Astr., 36, 266

Tutukov, A. A., Yungelson, L. R., \& Iben, I., Jr. 1992, ApJ, 386, 197

Verbunt, F., \& Zwaan, C. 1981, A\&A, 100, L7

Webbink, R. F. 1984, ApJ, 277, 355

Wheeler, J. C. 1991, in Evolutionary Processes in Interacting Binary Stars, ed. Y. Kondo et al. (Dordrecht: Kluwer), p. 225

Whelan, J., \& Iben, I., Jr. 1973, ApJ, 186, 1007

Woosley, S. E., \& Weaver, T. A. 1994, ApJ, 423, 371 